WellBeing International

WBI Studies Repository

12-1999

\title{
Scale-Model Comprehension by Chimpanzees (Pan troglodytes)
}

Valerie A. Kuhlmeier

The Ohio State University

Sarah T. Boysen

The Ohio State University

Kimberly L. Mukobi

The Ohio State University

Follow this and additional works at: https://www.wellbeingintlstudiesrepository.org/acwp_asie

Part of the Animal Studies Commons, Comparative Psychology Commons, and the Other Animal

Sciences Commons

\section{Recommended Citation}

Kuhlmeier, V. A., Boysen, S. T., \& Mukobi, K. L. (1999). Scale-model comprehension by chimpanzees (Pan troglodytes). Journal of comparative psychology, 113(4), 396.

This material is brought to you for free and open access by WellBeing International. It has been accepted for inclusion by an authorized administrator of the WBI Studies Repository. For more information, please contact wbisr-info@wellbeingintl.org.

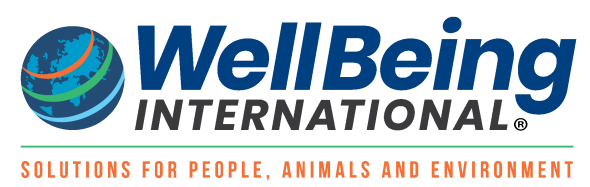




\title{
Scale-Model Comprehension by Chimpanzees (Pan troglodytes)
}

Valerie A. Kuhlmeier, Sarah T. Boysen, and Kimberly L. Mukobi

Ohio State University

\begin{abstract}
$\underline{\text { ABSTRACT }}$
The ability of chimpanzees (Pan troglodytes) to recognize the correspondence between a scale model and its real-world referent was examined. In Experiments 1 and 2, an adult female and a young adult male watched as an experimenter hid a miniature model food in 1 of 4 sites in a scale model. Then, the chimpanzees were given the opportunity to find the real food item that had been hidden in the analogous location in the real room. The female performed significantly above chance, whereas the male performed at chance level. Experiments 3 and 4 tested 5 adult and 2 adolescent chimpanzees in a similar paradigm, using a scale model of the chimpanzees' outdoor area. Results indicate that some adult chimpanzees were able to reliably demonstrate an understanding of the relationship between a scale model and the larger space it represented, whereas other subjects were constrained by inefficient and unsuccessful search patterns.
\end{abstract}

Flexible symbol use and comprehension have recently been a significant focus in the study of the cognitive abilities of chimpanzees (Pan troglodytes; e.g., Matsuzawa, 1985; Savage-Rumbaugh, 1986). Chimpanzees have demonstrated the capacity for understanding sophisticated representational concepts related to rudimentary language-like skills (e.g., Premack, 1971, 1986) as well as other representational systems, such as numbers (e.g., Boysen \& Berntson, 1989; Matsuzawa, 1985; Murofushi, 1997). However, in an early attempt to test chimpanzees' understanding of another representational relationship, juvenile chimpanzee subjects failed to recognize the correspondence between a scale model of a familiar room and the actual room (Premack \& Premack, 1983). However, important procedural details were not reported, making it difficult for one to compare these chimpanzees' difficulties with children's performance on similar tasks and also with the results of the present set of studies with chimpanzees. Human children show a striking age difference in understanding scale models (DeLoache, 1987, 1991, 1995). DeLoache reported that during a task in which children who witnessed a miniature toy being hidden in a model were asked to find the analogous full-size toy in a real room, 3-year-old children readily retrieved the real toy, whereas $2 \frac{1}{2} 2$-year-old children had difficulty with the task. The younger children's poor performances were not due to a memory failure for the original hiding site, because they were able to find the miniature toy when they were asked to do so after returning to the model (DeLoache, 1987, 1991).

DeLoache $(1987,1991,1995)$ explained the performance problems of the younger children as reflecting more limited abilities to form a "dual orientation" to the model. To solve the task, the children must be able to represent the model as both a tangible, real object and a symbol for something else. The dual- 
orientation hypothesis was supported by additional findings when the same children were presented with photographs of the hiding sites instead of the model. With photographs, both age groups were able to find the hidden toy. Thus, in situations in which the younger children (2⿺辶2-year-olds) had to form a dual orientation (scale-model task), their performance was poorer, whereas photographs were readily interpreted representationally (DeLoache, 1991). In contrast, the older children recognized the model as both symbol and object.

In light of the negative findings of Premack and Premack (1983) as well as the developmental differences noted by DeLoache (e.g., 1987, 1991), we designed the present study to explore the ability of chimpanzees to understand the topographic relationship between a scale model and its corresponding real-world space by using an approach closely modeled after DeLoache's (1987) research. The first study used a scale model of an indoor room that was very familiar to the 2 chimpanzee subjects, which were able to interact safely with Sarah T. Boysen outside of their home cage. A second scale model of a familiar outdoor enclosure was used in Experiment 3 and allowed all7 chimpanzees ( 5 adults and 2 adolescents) in the Ohio State University colony to be tested.

\section{Experiment 1}

Method

Subjects

Two chimpanzees (Pan troglodytes) housed at the Ohio State University Chimpanzee Center served as subjects. Both an adult female, Sheba (15 years old), and an adolescent male, Bobby (9 years old), had extensive prior experience on a range of cognitive tasks (e.g., Boysen, Berntson, Hannan, \& Cacioppo, 1996; Limongelli, Boysen, \& Visalberghi, 1995; Thompson, Oden, \& Boysen, 1997) and continued to participate in several ongoing cognitive studies at the time of the present study.

\section{Materials}

The chimpanzees were tested in a carpeted room ( $3.05 \mathrm{~m}$ wide $\times 5.18 \mathrm{~m}$ long) that was familiar to both subjects. Throughout testing, the room contained four furnishings: a blue metal cabinet, an artificial tree, a large blue plastic tub, and a brightly colored fabric-covered foam chair. A scale model that was one seventh the size of the room ( $44 \mathrm{~cm}$ wide $\times 74 \mathrm{~cm}$ long) was placed directly outside in an adjacent hallway. The model contained miniature versions of the furniture, carpet, and other permanent features of the room, including a large window facing an interior hallway in the real world. An actual aluminum can of soda and a miniature version of the can were used as hiding items.

\section{Procedure}

As described with children in DeLoache $(1987,1991)$, before any formal testing, each subject completed an orientation phase that consisted of three steps. First, the model was placed in the center of the actual room. The experimenter then displayed each miniature item next to the analogous full-size item and verbally and gesturally drew attention to the items. The chimpanzee was allowed to handle the miniature items as well as to explore the room. Next, the model was moved to the adjacent hallway directly outside of the room. As the chimpanzee watched, the experimenter hid the miniature can of soda behind the tree in the scale model. The experimenter and the chimpanzee then entered the full-size room, and the experimenter "hid" the real soda can behind the tree while the chimpanzee watched. Then, both the experimenter and the chimpanzee exited the room. At this point, the chimpanzee was allowed to reenter the room, was encouraged to locate the "hidden" soda, and was allowed to consume it after it was found behind the artificial tree (which both subjects did immediately). During a second orientation trial, the 
subjects witnessed the hiding of only the miniature food item in the model and were not allowed to watch as the experimenter hid the real soda can in the full-size room. When given the opportunity to search the real room, however, both chimpanzees were immediately successful in locating the hidden soda can.

All hiding events and test trials were videotaped for later analyses. For the hiding events and Retrieval 2 (see below), a color video camera (Quasar Palmcorder, Model VM565) was positioned in front of the model in the hall adjacent to the actual playroom. All test trials (Retrieval I) were recorded through the window of the room by using a color video camera (Sharp Jetzoom VHS, Model VL-L330U), which was positioned in the interior hallway. Thus, two sets of videotape recordings of all hiding events and retrievals allowed for a permanent visual record of all phases of the experiments. Responses also were recorded on data sheets by a second experimenter who observed all trials through a color monitor connected to the second video camera.

\section{Testing}

Formal testing consisted of a total of eight trials per chimpanzee, which included three phases: (a) the Hiding Event: The chimpanzee watched as the experimenter placed the miniature soda can in one of four hiding places in the model; the experimenter then displayed the real soda to the chimpanzee and moved into the real room to hide it while the chimpanzee remained in the hall outside the room; and after hiding the can, the experimenter returned to the chimpanzee in the hallway; (b) Retrieval 1: The chimpanzee was then allowed access to the room to search for the full-size can while the experimenter remained in the hall; and (c) Retrieval 2: To test the chimpanzee's ability to remember the hiding site in the model, the chimpanzee was then led back to the model and encouraged to indicate (by pointing or touching) where the object had been hidden.

Only correct retrievals that occurred on the first search attempt were considered in subsequent data analyses. For both chimpanzees, eight trials were completed over 4 days (two trials per day), with each site used twice to minimize interference effects. Trial order was randomly assigned. After the eight standard scale-model trials were completed, each chimpanzee received eight more trials during which only the object that represented the hiding site itself (the miniature chair, cabinet, tub, or tree) was presented during the hiding event; the entire model was not present.

\section{$\underline{\text { Results }}$}

For all experiments, we used binomial analyses with an alpha level set at .05, with chance level at .25. During the first 8 trials of Experiment 1, which used the entire scale model and its contents, only 1 chimpanzee (Sheba) was able to find the real soda can at a level significantly above chance (7 out of 8 correct responses, or $88 \%$; Figure 1). The performance of the younger male (Bobby) reached statistical significance only during Retrieval 2, when he was required to indicate the original hiding place of the item in the scale model. Bobby was unsuccessful at finding the actual soda can in the real room. During the second set of trials in Experiment 1, when only one of the miniature objects representing the four locations was presented on a given trial, again, only Sheba performed at a level above chance (5 out of 8 correct responses, or $63 \%)$.

\section{Discussion}

The performance of 1 chimpanzee subject (Sheba) suggested that she was able to use the model as a source of information for the location of the hidden can in the real room. In seven of eight trials, Sheba entered the room, moved directly to the hiding site, and found the soda. Not surprisingly, during the simplified task in which only the miniature representation of the hiding site was shown, she also was able to readily find the real can in the full-size test room. Given Bobby's initial success during the orientation 
trials (he was able to find the can hidden in the real room with no assistance from the experimenter) and his strong performance on Retrieval 2, it was surprising that he was not able to reliably choose the correct site in the real room during formal testing. His search attempts showed strong evidence of motor perseveration. On all but one of the trials, he entered the room and immediately searched under the blue tub. When unsuccessful, he then routinely searched the other sites in the room until he found the soda can, following a clockwise pattern around the room as he visited each of the other three locations. Even in the simplified trials using only the miniature hiding site, Bobby continued to show the same type of perseverative response patterns. Thus, the younger subject failed to use knowledge gained from observing the item being hidden in the model and instead relied on a fairly rigid spatial search pattern.

Figure 1. Percentage of correct responses for Sheba and Bobby for both the standard scale model and the individual sites task in Experiment 1. * $p<.05$.

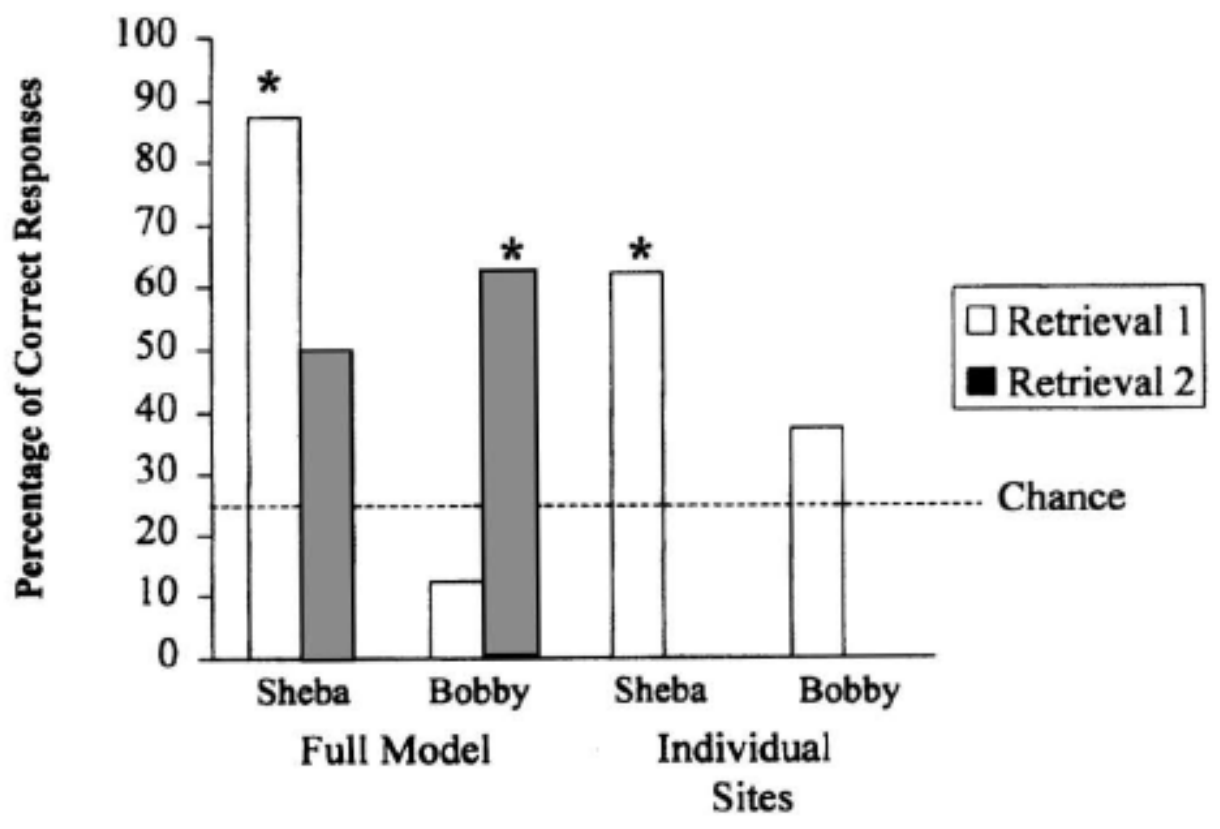

\section{Experiment 2}

The second experiment was designed to examine whether chimpanzees could use knowledge gained from photographs of an adjacent room to locate a desired object hidden in the room. Of particular interest was whether Bobby, the subject who had failed previous tests with the scale model, would be able to perform better with a photographic representation of the room and thus show evidence of the "picture superiority" effect reported by DeLoache $(1987,1991)$ for younger human participants.

\section{$\underline{\text { Method }}$}

Materials

The full-size room and its furnishings remained unchanged from the description for Experiment 1. However, color photographs $(20.0 \mathrm{~cm}$ wide $\times 27.5 \mathrm{~cm}$ long) of each of the four items in the room and a color panoramic photograph $(20.0 \mathrm{~cm}$ wide $\times 27.5 \mathrm{~cm}$ long) of the entire room were used instead of the scale model and its contents. The four photographs were mounted in a row, approximately $60 \mathrm{~cm}$ from 
the floor, on the wall outside the room. As in Experiment 1, a can of soda was hidden in the full-size room during each trial.

\section{Procedure}

Four-photographs condition. Each test trial had two phases: (a) the Hiding Event: The chimpanzee watched as the experimenter displayed the real soda can and pointed to the photograph of the correct hiding site; the experimenter then entered the room, leaving the chimpanzee in the hall; hid the can; and returned to the hall and (b) Retrieval: The chimpanzee was then allowed to enter the room and search for the soda can. As in Experiment 1, only the chimpanzees' first search attempts were considered in subsequent analyses. For this and all other conditions, eight trials (two trials per site) were completed over 4 days, with site order randomly determined.

Individual-photographs condition. In the second condition, the chimpanzees saw only the photograph of the site used on that particular trial; the other three photographs were out of view. All other procedures remained consistent with those described for the four-photographs condition.

Panoramic-photograph condition. In the third condition, a panoramic photograph of the entire room was shown to the subjects. During the hiding event, the experimenter pointed to the appropriate site in the panoramic photograph before hiding the can in the real room (no verbal labels for the sites were used as the experimenter pointed to the sites in the photograph because the chimpanzees understood a great deal of spoken English).

Figure 2. Percentage of correct responses for Sheba and Bobby during the three conditions of the photograph task in Experiment 2. * $p<.05$.

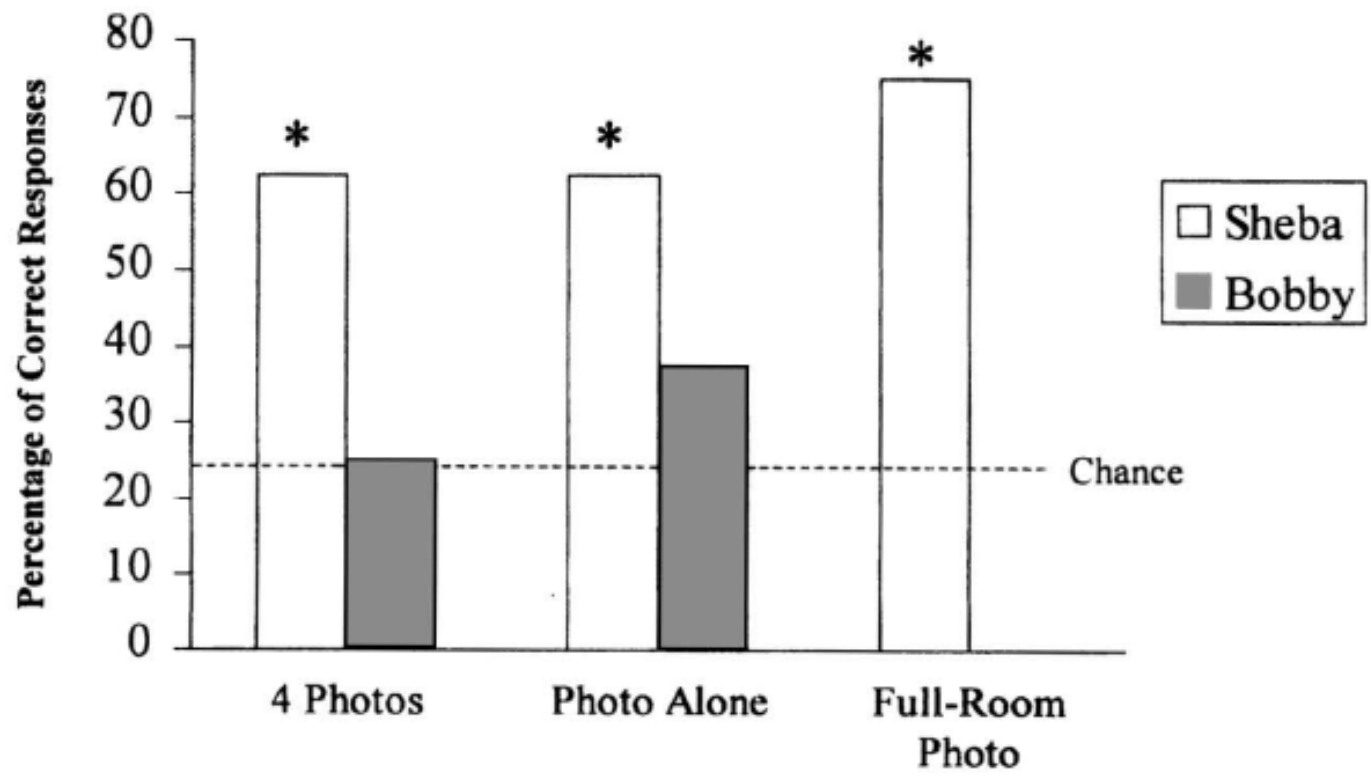

$\underline{\text { Results }}$

The adult female subject (Sheba) located the soda can in the full-size room at levels significantly above chance during all three conditions of the photograph task (four photos: 5 out of 8 correct respones, or 
$63 \%$; individual photos: 5 out of 8 correct responses, or 63\%; and panoramic photos: 6 out of 8 correct responses, or 75\%; Figure 2). Bobby, however, failed under all three conditions. He did not respond at levels significantly above chance either when the photo of the hiding sites was indicated from among all four displayed or when only a single photograph representing the hiding site was presented, and he failed to get a single trial correct when the panoramic photograph of the playroom was used.

\section{Discussion}

During Experiment 2, Sheba's performance indicated that she was able to use photographs of either the individual sites or the entire room as a source of information regarding the location of the hidden item in the real room. DeLoache's $(1987,1991)$ picture superiority effect was not seen in the present study with the younger chimpanzee subject that also had difficulty with the scale-model version of the task. When the symbolic modality was changed to photographs, Bobby was unable to reliably retrieve the hidden can and, instead, displayed the same perseverative response pattern observed in Experiment 1, during which he persisted at searching the same incorrect site for several trials in a row and then followed a clockwise search pattern from site to site.

\section{Experiment 3}

The results of Experiments 1 and 2 raised several additional questions related to the ability of chimpanzees to understand the perceptual correspondence between scale models and their referents. Among these questions was whether the 2 chimpanzees' performance differences in Experiments 1 and 2 were related to individual, age, or sex differences. To test all adult chimpanzees in the group, most of which were no longer handled outside of their cages, a scale model of one portion of their outdoor play area was constructed. By testing the adult group in their outdoor enclosure and a corresponding scale model, we were able to examine the performance of 5 additional subjects using DeLoache's (1987) task. We were especially interested in the types of search strategies we might see used, particularly by unsuccessful subjects, in order to address additional questions raised by Bobby's inability to use the spatial information represented by the model in the first experiment.

\section{Method}

\section{Subjects}

Seven chimpanzees (3 females: Abby, Sarah, and Sheba; 4 males: Bobby, Darrell, Digger, and Kermit), including Bobby and Sheba, who had participated in Experiments 1 and 2, served as subjects in Experiment 3. All but 2 of the subjects (Digger and Abby) had previous experience on a range of cognitive tasks (e.g., Boysen, 1994; Limongelli et al., 1995; Thompson et al., 1997).

\section{Materials}

The chimpanzees were tested in an outdoor enclosure $(3.25 \mathrm{~m}$ wide $\times 5.48 \mathrm{~m}$ long) with a concrete floor covered with large nugget pine bark. The scale model of the outdoor area was one seventh the size of the real enclosure. Both the enclosure and the model contained four hiding sites. The sites in the enclosure were indicated by large play items that were familiar to all of the chimpanzees and included a small rubber tire from a garden tractor, a plastic barrel, a children's green plastic teeter-totter that was shaped like an alligator, and a red sandbox shaped like a large crab. The items in the model were perceptually similar in color and shape but were 1:7 scale miniatures of the larger items. The model toys were constructed of clay, plastic, and cardboard and were painted to match the full-size objects. Both the large and the miniature items were arranged in four positions in the enclosure -and the model, respectively, and remained in the same locations throughout Experiment 3. A plastic bottle filled with fruit juice was hidden 
in the outdoor enclosure on each trial, and a miniature version made from orange-colored clay was used for hiding in the scale model.

\section{Procedure}

Orientation. In the first phase, an experimenter brought the scale model into the testing enclosure while a chimpanzee remained in an adjacent cage. This arrangement allowed visual access for the chimpanzee to both the testing enclosure and the scale model. The experimenter faced the subject and displayed each item in the model next to the analogous full-size item. In the second phase, the experimenter hid the miniature juice bottle in the model and then "hid" the real bottle in the analogous location in the enclosure in full view of the chimpanzee. The experimenter took the model and exited the enclosure, and the chimpanzee was then allowed to enter and locate the "hidden" juice bottle.

Testing. Before formal testing began, a canvas tarp was hung on the inside wire mesh walls of the testing cage; these barriers allowed for the juice bottle to be hidden in the enclosure without the chimpanzees witnessing the event from the adjacent cages. Otherwise, the testing conditions were similar to those used in Experiments 1 and 2. The subjects were tested individually and watched as the experimenter placed a miniature version of the juice bottle in one of the four locations (tire, barrel, teeter-totter, or sandbox) in the model. Next, the chimpanzee was given access to the outdoor play yard, where the real juice bottle had been hidden, and was allowed to search the enclosure until he or she located the bottle (Retrieval). Each chimpanzee completed 20 trials, with each hiding site used five times; trial order was randomized for each subject. Only the Hiding Event and Retrieval 1 were completed. Because of the small size of the wire mesh of the caging, it was virtually impossible for the chimpanzees to specifically indicate (e.g., by pointing) the location of the hidden object in the scale model because of the size of the chimpanzees' hands and the depth of the three-dimensional model. Therefore, it was not possible to have the subjects complete Retrieval 2 for this experiment.

\section{$\underline{\text { Results }}$}

Three of the 7 chimpanzees successfully located the juice bottle in the outdoor enclosure at levels that were significantly above chance (Sheba: 11 out of 20 correct responses, or 55\%; Sarah: 13 out of 20 correct responses, or 65\%; and Darrell: 11 out of 20 correct responses, or 55\%; Figure 3). Of the unsuccessful subjects, all 4 chimpanzees showed a preference for searching a particular site first (Position 1, the tire) on each trial (Abby: 16 out of 20 trials, or 80\%; Bobby: 18 out of 20 trials, or 90\%; Digger: 11 out of 20 trials, or 55\%; and Kermit: 20 out of 20 trials, or 100\%). However, 1 subject (Abby) chose the correct site in her second search attempt (12 out of 14 searches, or $86 \% ; p<.05$ ) significantly above chance $(p=.33)$. The other unsuccessful subjects did not achieve comparable performance levels; instead, after their initial incorrect choice (Position 1), they usually moved to the site that was immediately adjacent. If third choices were made, they were sites that were in the same clockwise direction (Position 3 or 4).

\section{Discussion}

The results with 2 other adult chimpanzee subjects were comparable to Sheba's performance with the indoor model (Experiment 1). The 3 successful subjects witnessed the hiding event in the model, entered the enclosure, went directly to the correct site, and retrieved the hidden juice. The choice patterns of the unsuccessful chimpanzees (with the exception of 1 adult female) suggested that they were relying on a search pattern of exploring each of the sites in the enclosure in a clockwise manner until locating the hidden juice bottle. The performance of 1 adult female (Abby) consisted of routinely searching at Position 1 and then immediately moving to the correct site on her second choice. Her performance suggested that 
she may have had some rudimentary understanding of the relationship between the model and the actual enclosure but was limited by some perceptual and attentional demands of the task. Similarly, the other 3 unsuccessful subjects, which included 1 adult male and 2 adolescent males, followed the same perseverative, clockwise, spatial routine, resulting in suboptimal performance.

Figure 3. Percentage of correct responses for all chimpanzees during Experiment 3. ${ }^{*} p<.05$.

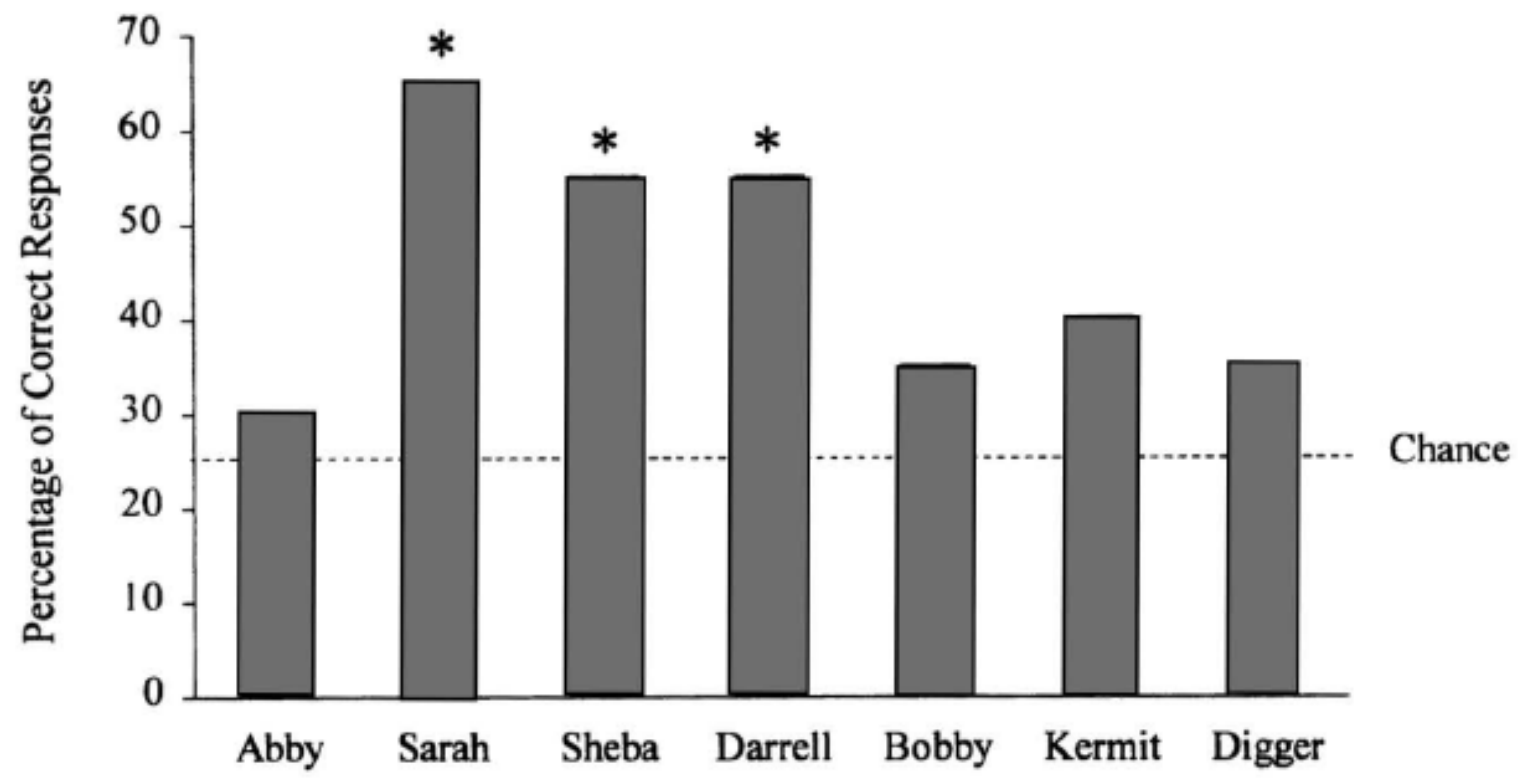

\section{Experiment 4}

Method

Subjects

In an attempt to disrupt the observed rigid search patterns displayed by the unsuccessful subjects in the three previous experiments, we retested all 7 chimpanzees by using a slightly modified paradigm.

\section{Procedure}

During Experiment 4, all of the toys in the enclosure as well as their miniature counterparts in the model were moved to new positions on each trial. All other procedures were comparable to those used in Experiment 3. For each subject, the hiding site and the toy position were counterbalanced such that each toy was used as a hiding site twice and each of the four different spatial positions served as the hiding site two times. The subjects completed a total of eight trials.

\section{$\underline{\text { Results }}$}

Three chimpanzees performed at levels significantly above chance under these test conditions (Figure 4), including Sheba (5 out of 8 trials, or $63 \%$ ) and Sarah (5 out of 8 trials, or $63 \%$ ), who were successful on the previous task, and also Abby (5 out of 8 trials, or $63 \%$ ), who had failed to reach significant performance levels on the basis of her initial choice during Experiment 3. Response patterns of the 4 
unsuccessful chimpanzees were analyzed again. There was a significant preference for Position 1 (Bobby: 7 out of 8 trials, or $88 \%$; Darrell: 6 out of 8 trials, or $75 \%$; Digger: 8 out of 8 trials, or $100 \%$; and Kermit: 6 out of 8 trials, or 75\%), and similar to Experiment 3 (when the 4 toy sites remained in fixed positions from trial to trial), there was a high frequency of searching at Position 2 for their second choice and then continuing the search in the same clockwise direction.

\section{Discussion}

During Experiment 4, when the potential hiding sites were moved on each trial and the location of the hidden juice bottle was randomly assigned on a given trial, 3 adult chimpanzees performed successfully at levels that were significantly above chance. Abby's demonstrated competence under these test conditions was particularly interesting given her unsuccessful performance on Experiment 3 when the four sites were fixed. We had hypothesized that if the sites were moved on each trial, this would serve to disrupt the rigid routine that some of the subjects were exhibiting, beginning with the initial search at Position 1 and moving in a clockwise pattern around to all four sites until the hidden juice was discovered. For Abby, moving the sites on each trial did facilitate her performance, such that her results were comparable to those of both Sheba and Sarah. However, changing the location of the four sites did not appear to serve the same facilitative effect for the 2 youngest subjects, which included the 2 adolescent males. Thus, with the exception of greatly improved performance by Abby and some enhancement of Kermit's score, in general, moving the sites between trials did not significantly affect the spatial search patterns used by all 4 unsuccessful subjects.

Figure 4. Percentage of correct responses for all chimpanzees during Experiment 4. ${ }^{*} p<.05$.

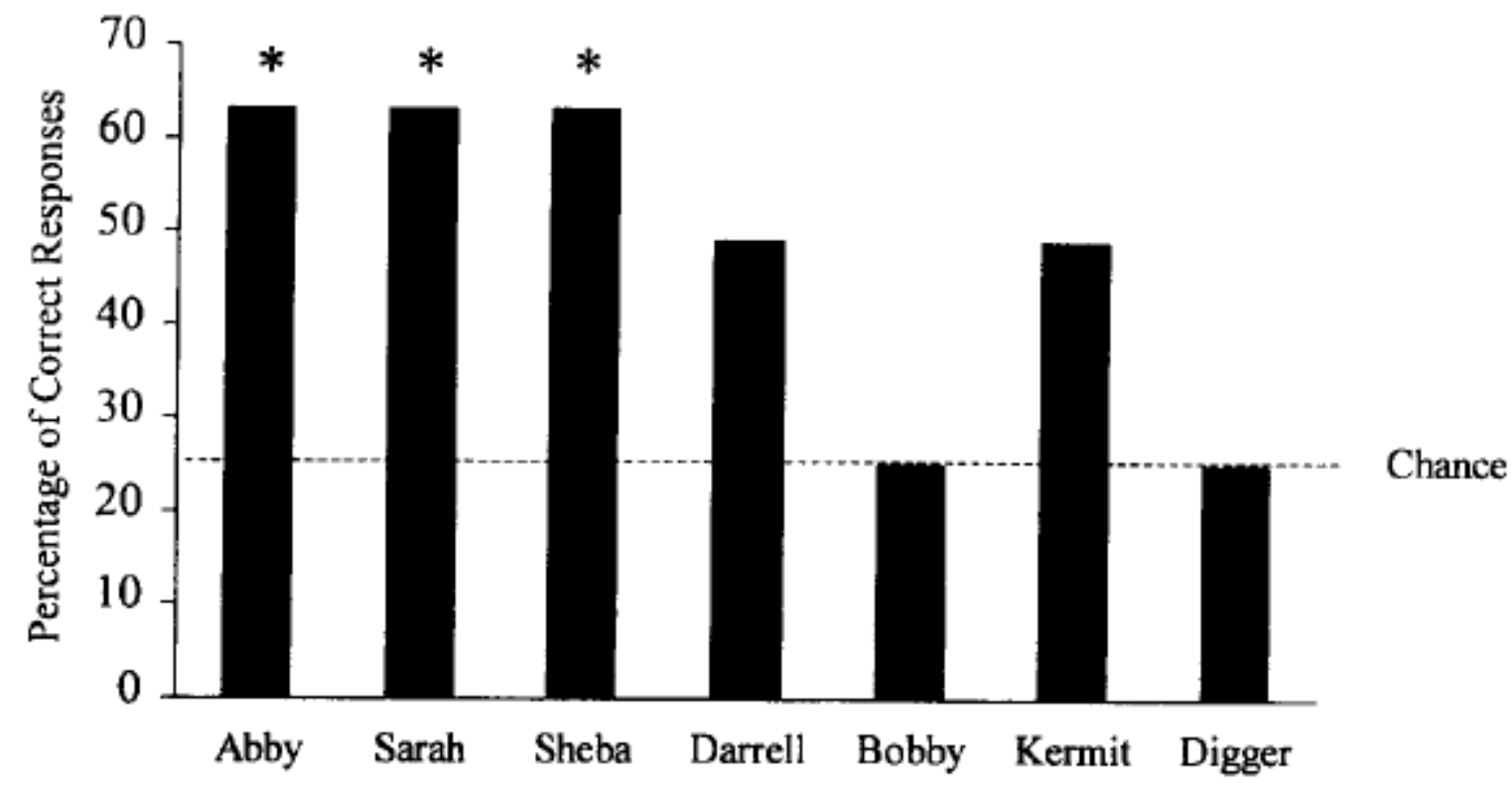

\section{$\underline{\text { General Discussion }}$}

Our results replicate and extend the innovative studies of similar skills in children by DeLoache (e.g., 1987, 1991) to a nonverbal, nonhuman species, the chimpanzee (Pan troglodytes). These four experiments provide evidence that, in contrast to the findings of Premack and Premack (1983), 
chimpanzees have the cognitive capacity to recognize the relationship between a scale model or photographs and representations of the corresponding real-world space and then can appropriately apply that information. The chimpanzees were able to approach the model as something other than a toy-like object in their environment and, instead, were able to recognize its representational function.

However, for some chimpanzees in our study, the task demands associated with the scale-model comprehension task were difficult, as evidenced by the problems some subjects had with the task. It is unclear whether their poor performance in most of the experiments was due to an inability to form a dual orientation, as suggested by DeLoache (1987) for her younger participants, who also had limited success with the scale-model task. Thus, according to this hypothesis, the chimpanzees were unable to view the model as an object in and of itself and as a symbolic representation of the real room. Another possibility is the failure to inhibit a somewhat rigid response pattern, which was not an optimal strategy for solving the task, particularly for the adolescent males.

Some evidence points to other possible interpretations. For example, in Experiment 1 , in the case of Bobby's limited performance, if his difficulty to efficiently solve the task stemmed from failure to form a dual orientation to the model, it is possible that his performance might have improved when photographs were used. However, use of photographs did not facilitate success for him on the task, and he continued to demonstrate perseverative search patterns. It is nevertheless possible that he was able to form a dual orientation to the model but he was unable to sufficiently inhibit a highly prepotent response strategy that had little flexibility. Such a spatial search pattern, although inefficient, ultimately did lead to the otherwise unsuccessful subjects finding the soda in all four experiments, because it was not possible to stop a trial to remove the soda once the trial began if the subjects were wrong in their initial choice of locations. Thus, the subjects who failed to initially choose the correct site may have had an underlying understanding of the representational nature of the model but were unable to inhibit both the development and the maintenance of the spatial motor routine on subsequent trials because the routine did ultimately result in reward. It is also possible that the attentional demands of the task, which were considerable, exceeded the capacities of some of the subjects, as individual differences in cognitive style and learning capacities have been previously noted across the chimpanzee group (e.g., Boysen, 1994).

After Experiment 3, we attempted to further examine the error patterns that were repeatedly observed in the subjects who were unsuccessful and to further explore the possible differences between the chimpanzees with reported performances by the younger children in studies by DeLoache (1991). The topography of the spatial search patterns exhibited by the chimpanzee subjects had not been reported for children. Instead, DeLoache noted that unsuccessful children most frequently searched at the site where the toy had been hidden on the previous trial. The chimpanzees' pattern entailed moving in a clockwise rotation from site to site until the hidden soda can was discovered. These perseverative search strategies survived numerous manipulations of the original scale-model task, including conditions during which the miniature items were presented individually and when photographs were used (Bobby, Experiments 1 \& 2), as well as when the toy sites were moved between trials (all unsuccessful chimpanzees, Experiments $3 \& 4)$.

During Experiment 3, the performances of Abby and Darrell and, to some extent, Kermit suggested that these chimpanzees may have been acquiring some limited information from the hiding event in the model. Abby's correct choices when the sites were fixed (Experiment 3) and her subsequent success when the sites were moved (Experiment 4) suggest that she came to recognize the correspondence between the model and the locations in the outdoor enclosure, but until the sites were moved on each trial, she was unable to disengage from repeatedly searching in the same initial location (Position 1). Darrell's performance when the sites were fixed (Experiment 3) suggests that he also was able to understand the representational relationship between the model and the enclosure. However, when the 
sites were moved (Experiment 4), he was unable to optimally use information about the model, and his performance decreased to a level approaching chance, perhaps because he was unable to inhibit using this rigid search strategy. Kermit's overall performance when the sites remained fixed was slightly better than that of both Bobby and Digger, but he still was correct on only $40 \%$ of trials. When the sites were moved, however, his performance increased to $50 \%$, just one trial short of statistical significance and at a level equivalent to Darrell's. As suggested for Darrell, Kermit may have been gleaning some information from the model, but his spatial perseveration pattern may have overwhelmed any potential understanding of the model's symbolic function.

Throughout all four experiments, the strongest performances were exhibited by the adult female subjects. The majority of the males tested, particularly the 2 adolescents, more frequently exhibited a suboptimal search pattern that did not contribute to successful performance. Because the Ohio State University chimpanzee colony does not currently have any adolescent females of comparable age to Digger and Bobby, it would be interesting to determine how subjects in this cohort would perform on the scale-model task. It is possible that success by chimpanzees, similar to the studies with children, is age-dependent, with a significant delay in demonstrated competence by chimpanzees until they are well into adulthood. Consequently, the previous negative results reported by Premack and Premack (1983) may have been due to the young ages of the chimpanzees they tested.

Overall, the results from the present series of studies exploring scale-model comprehension by chimpanzees provide evidence that although some chimpanzees reliably show an ability to understand the relationship between a scale model and its real-world counterpart, there may be behavioral and cognitive constraints faced by chimpanzees during testing on this task that children may not experience. The demonstrated failures by some subjects in these studies were the result of the implementation of rigid spatial search patterns that may have resulted from their inability to represent the model as a symbol, failure to inhibit prepotent behavioral response strategies that might otherwise have been advantageous, or task demands that created attentional deficits or taxed attentional capacity in some subjects. Further studies are necessary to address additional hypotheses generated by the experiments and to investigate the potential parameters that facilitate successful interpretation of a scale model by chimpanzees and those that preclude optimal performance in others, particularly adolescent males.

\section{References}

Boysen, S. T. (1994). Individual differences in the cognitive abilities of chimpanzees. In R. W. Wrangham, W. C. McGrew, F. B. M. de Waal, \& P. G. Heltne (Eds.), Chimpanzee cultures (pp. 335-350). Cambridge, MA: Harvard University Press.

Boysen, S. T., \& Berntson, G. G. (1989). Numerical competence in a chimpanzee (Pan troglodytes). Journal of Comparative Psychology, 103, 23-31.

Boysen, S. T., Berntson, G. G., Hannan, M. B., \& Cacioppo, J. T. (1996). Quantity-based interference and symbolic representations in chimpanzees (Pan troglodytes). Journal of Experimental Psychology: Animal Behavior Processes, 22, 76-86.

DeLoache, J. S. (1987, December 11). Rapid change in the symbolic functioning of very young children. Science, 238, 1556-1557.

DeLoache, J. S. (1991). Symbolic functioning in very young children: Understanding of pictures and models. Child Development, 62, 736-752.

DeLoache, J. S. (1995). Early symbol understanding and use. In D. L. Medin (Ed.), The psychology of learning and motivation (pp. 65-114). San Diego, CA: Academic Press.

Limongelli, L., Boysen, S. T., \& Visalberghi, E. (1995). Comprehension of cause-effect relations in a toolusing task by chimpanzees (Pan troglodytes). Journal of Comparative Psychology, 109,47-51.

Matsuzawa, T. (1985) Use of numbers by a chimpanzee. Nature, 315,57-59. 
Murofushi, K. (1997). Numerical matching behavior by a chimpanzee (Pan troglodytes): Subitizing and analogue magnitude estimation. Japanese Psychological Research, 39, 140-153.

Premack, D. (1971, May 21). Language in a chimpanzee? Science, 172, 808-822.

Premack, D. ( 1986). Gavagai. London: Cambridge University Press.

Premack, D., \& Premack, A. J. (1983). The mind of an ape. New York: Norton.

Savage-Rumbaugh, E. S. (1986). Ape language: From conditioned response to symbol. New York: Columbia University Press.

Thompson, R. K. R., Oden, D. L., \& Boysen, S. T. (1997). Language-naive chimpanzees (Pan troglodytes) judge relations between relations in an abstract matching task. Journal of Experimental Psychology: Animal Behavior Processes, 23, 31-43. 